\title{
CONSTRAINTS FOR THE NUCLEAR SEA QUARK DISTRIBUTIONS FROM THE DRELL-YAN PROCESS AT THE SPS
}

\author{
K.J. Eskola ${ }^{\mathrm{a}, \mathrm{d}}$, V.J. Kolhinen ${ }^{\mathrm{a}}$, C.A. Salgado ${ }^{\mathrm{b}}$ and R.L. Thews泡 \\ a Department of Physics, University of Jyväskylä, \\ P.O.Box 35, FIN-40351 Jyväskylä, Finland \\ b Laboratoire de Physique Théoriqu币, Université de Paris XI, \\ Bâtiment 211, F-91405 Orsay Cedex, France \\ c Department of Physics, University of Arizona, \\ Tucson, Arizona 85721, USA \\ d Helsinki Institute of Physics, \\ P.O. Box 9, FIN-00014 University of Helsinki, Finland
}

\begin{abstract}
Nuclear modifications to the Drell-Yan dilepton production cross sections in $\mathrm{p}+A$ and $A+A$ collisions in the leading twist approximation are caused by nuclear effects in the parton distributions of bound nucleons. For non-isoscalar nuclei, isospin corrections must also be considered. We calculate these effects for $\mathrm{p}+A$ and $\mathrm{Pb}+\mathrm{Pb}$ collisions at CERN SPS energies. Our goal is to place constraints on nuclear effects in sea quark distributions in the region $x \gtrsim 0.2$. We show that the net nuclear corrections remain small for $\mathrm{p}+A$ collisions at $E_{\text {lab }}=450 \mathrm{GeV}$. However, in $\mathrm{Pb}+\mathrm{Pb}$ collisions at $E_{\text {lab }}=158 \mathrm{AGeV}$, effects of $\gtrsim 20 \%$ are predicted at large $M$. The data collected by the NA50 collaboration could thus be used to constrain the nuclear effects in the sea quark distributions in the region of the EMC effect, $x \gtrsim 0.3$.
\end{abstract}

\footnotetext{
${ }^{1}$ kari.eskola@phys.jyu.fi

${ }^{2}$ vesa.kolhinen@phys.jyu.fi

${ }^{3}$ carlos.salgado@th.u-psud.fr

${ }^{4}$ thews@physics.arizona.edu

${ }^{5}$ Laboratoire associé au Centre National de la Recherche Scientifique - URA D0063.
} 


\section{Introduction}

In this work, we study perturbatively calculable QCD processes in high energy $\mathrm{p}+A$, $A+A$ and $A+B$ collisions. In the search for the Quark-Gluon Plasma (QGP) in ultrarelativistic heavy ion collisions, these inclusive hard processes provide reference cross sections for detecting specific signatures of the QGP, such as suppression of heavy quarkonia, production of thermal dileptons and photons, and energy losses of jets.

At high energies the inclusive differential cross sections of hard processes in nuclear collisions can be computed (in leading twist approximation) from a factorized form consisting of nuclear parton distributions and partonic cross sections. At this level all nuclear effects are contained in the nuclear parton distributions, which obey the Dokshitzer-Gribov-Lipatov-Altarelli-Parisi (DGLAP) evolution equations of perturbative QCD (pQCD) 11. At higher orders in pQCD, absorption of $(1 / \varepsilon)$ singularities into definitions of parton distributions is scheme-dependent (usually $\overline{M S}$ ), which retains the same universality properties as in the case of hard processes for free nucleon scatterings. Consequently, the same nuclear parton distributions can be used to compute different hard scattering cross sections in nuclear collisions. This is the obvious motivation to perform a consistent DGLAP analysis of nuclear parton distributions, as done in [2, 3, 4].

Symbolically, the inclusive hard scattering cross sections for producing a particle $c$ in a collision of nuclei $A$ and $B$ can be written as

$$
\begin{gathered}
d \sigma\left(Q^{2}, \sqrt{s}\right)_{A B \rightarrow c+X}=\sum_{i, j=q, \bar{q}, g}\left[Z_{A} f_{i}^{p / A}\left(x_{1}, Q^{2}\right)+\left(A-Z_{A}\right) f_{i}^{n / A}\left(x_{1}, Q^{2}\right)\right] \otimes \\
\otimes\left[Z_{B} f_{j}^{p / B}\left(x_{2}, Q^{2}\right)+\left(B-Z_{B}\right) f_{j}^{n / B}\left(x_{2}, Q^{2}\right)\right] \otimes d \hat{\sigma}\left(Q^{2}, x_{1}, x_{2}\right)_{i j \rightarrow c+x}
\end{gathered}
$$

where $\hat{\sigma}\left(Q^{2}, x_{1}, x_{2}\right)_{i j \rightarrow c+x}$ is the perturbatively calculable cross section at a large momentum (or mass) scale $Q \gg \Lambda_{Q C D} \sim 200 \mathrm{MeV}, x_{1,2} \sim Q / \sqrt{s}$ are the fractional momenta, $f_{i}^{p(n) / A}\left(x_{1}, Q^{2}\right)$ is the distribution of parton species $i$ in a proton (neutron) of the nucleus $A$, and correspondingly $f_{j}^{p(n) / B}$ is that for the nucleus $B$. The number of protons in $A(B)$ is denoted by $Z_{A}\left(Z_{B}\right)$. For isoscalar nuclei, the parton distributions of bound neutrons are obtained through isospin symmetry (as in the case of unbound nucleons), $f_{u(\bar{u})}^{n / A}=f_{d(\bar{d})}^{p / A}$ and $f_{d(\bar{d})}^{n / A}=f_{u(\bar{u})}^{p / A}$. This is expected to be a good approximation for non-isoscalar nuclei as well. Therefore, one may formulate the studies of nuclear parton distributions simply in terms of those in bound protons, which are denoted here as $f_{i}^{p / A} \equiv f_{i / A}$. It is convenient to define the nuclear effects in parton distributions in terms of the ratio of the distribution of the parton species $i$ in a bound proton to that in a free proton,

$$
R_{i}^{A}\left(x, Q^{2}\right) \equiv \frac{f_{i}^{p / A}\left(x, Q^{2}\right)}{f_{i / p}\left(x, Q^{2}\right)}
$$


Information on the nuclear parton distributions is primarily obtained by deep inelastic lepton-nucleus scattering (DIS) [5]-13] and by Drell-Yan (DY) dilepton production in proton-nucleus collisions 114, 15. The nuclear structure functions $F_{2}^{A}\left(x, Q^{2}\right)$, measured in DIS [5]-13] differ from those in free nucleons. The ratios $R_{F_{2}}^{A} \equiv \frac{1}{A} F_{2}^{A} / \frac{1}{2} F_{2}^{\mathrm{D}}$, where deuterium $D$ approximates an average free nucleon, show clear and systematic deviations from unity in various regions of Bjorken- $x$ : shadowing $\left(R_{F_{2}}^{A} \leq 1\right)$ at $x \lesssim 0.1$, antishadowing $\left(R_{F_{2}}^{A} \geq 1\right)$ at $0.1 \lesssim x \lesssim 0.3$, EMC effect $\left(R_{F_{2}}^{A} \leq 1\right)$ at $0.3 \lesssim x \lesssim 0.7$, and Fermi motion $\left(R_{F_{2}}^{A} \geq 1\right)$ as $x \rightarrow 1$ and beyond. The New Muon Collaboration (NMC) highprecision measurements of the $F_{2}$ structure function ratios for tin vs. carbon, $F_{2}^{\mathrm{Sn}} / F_{2}^{\mathrm{C}}$ [9] have also revealed a dependence on the virtuality scale $Q^{2}$ at small values of $x$. These measured modifications of nuclear structure functions directly imply modifications of parton distributions in bound nucleons.

Just as in the QCD-improved parton model (in lowest order) the structure functions can be written in terms of parton distributions,

$F_{2}^{A}\left(x, Q^{2}\right)=\sum_{q} e_{q}^{2}\left\{Z\left[x f_{q}^{p / A}\left(x, Q^{2}\right)+x f_{\bar{q}}^{p / A}\left(x, Q^{2}\right)\right]+(A-Z)\left[x f_{q}^{n / A}\left(x, Q^{2}\right)+x f_{\bar{q}}^{n / A}\left(x, Q^{2}\right)\right]\right\}$

where $q$ is the quark flavour and $e_{q}$ is the corresponding charge. In the DGLAP analysis of nuclear parton distributions [2, 3, 4] it is assumed that the distributions $f_{i}^{p / A}$ are factorizable at a sufficiently large initial scale, $Q_{0} \gg \Lambda_{Q C D}$. Once the input distributions are given at $Q_{0}^{2}$ and at $x \geq x_{m i n}$, their evolution is predicted by the DGLAP equations at $Q \geq Q_{0}^{2}$ and $x \geq x_{\text {min }}$. As a result, the ratios $R_{i}^{A}\left(x, Q^{2}\right)$ depend both on $x$ and $Q^{2}$. In analogy with the global analyses of the free parton distributions, the key problem is then to determine the (nonperturbative) initial distributions $f_{i}^{p / A}\left(x, Q_{0}^{2}\right)$. To constrain these, further information is needed. This is provided by the DIS measurements mentioned above (NMC [6]- 9], SLAC [10], E665 [11, 12]) and by the Drell-Yan data from the E772 and E866 collaborations in $\mathrm{p}+A$ collisions [14, 15]. In addition, conservation of momentum and baryon number serve as further constraints. We emphasize that the measured $Q^{2}$ dependence of the ratio $F_{2}^{\mathrm{Sn}} / F_{2}^{\mathrm{C}}[9]$ is also reproduced very well by the DGLAP evolution [3].

In the DGLAP analysis [3] of the nuclear parton distributions the nuclear effects were expressed in terms of free parton distributions which were assumed to be known, i.e. obtained from a set of distributions such as CTEQ, GRV, MRS etc. The absolute distributions from different sets of free parton densities may differ by a fairly large factor 9 and, consequently, these differencies will be reflected in the absolute nuclear parton distributions as well. The ratios $R_{i}^{A}\left(x, Q^{2}\right)$, however, vary only within a few percent from set to set, as shown in 4 . Therefore, for computing hard processes in nuclear collisions with nuclear effects in the parton distributions, it is a good approximation to

\footnotetext{
${ }^{6}$ ideally of course, there would be only one best set $\left\{f_{i}\left(x, Q^{2}\right)\right\}$
} 
use universal ratios $R_{i}^{A}\left(x, Q^{2}\right)$ which are independent of specific free parton densities. A parametrization of $R_{i}^{A}\left(x, Q^{2}\right)$, "EKS98", was prepared in [4] for general use, and it is available in [16] and now also in the the CERN PDFLIB library of parton densities [17].

Some uncertainties, however, remain in the determination of the nonperturbative input distributions at $Q_{0}^{2}$. In this paper, we will focus on constraining uncertainties in the input sea quark distributions in the region $x \gtrsim 0.2 . .0 .3$, i.e. approaching the region of the EMC effect, where the ratios $R_{F_{2}}^{A}$ measured in DIS are dominated by valence quarks. Our goal here is to study to what extent the NA50 Drell-Yan data for $\mathrm{p}+\mathrm{p}$ and $\mathrm{p}+A$ collisions at $E_{\mathrm{lab}}=450 \mathrm{GeV}$ and $\mathrm{Pb}+\mathrm{Pb}$ collisions at $E_{\mathrm{lab}}=158 \mathrm{AGeV}$ at the CERN-SPS [18] can be used to constrain the EMC effect for the input distributions of the nuclear sea.

\section{General properties of nuclear corrections}

Let us first discuss in some detail how available data and sum rules constrain the input nuclear parton distributions, or equivalently the nuclear modifications $R_{i}^{A}\left(x, Q_{0}^{2}\right)$, in different regions of $x$ in the DGLAP approach [3]. Since the DGLAP analysis a perturbative, the scale evolution must be limited to the region $Q \geq Q_{0} \sim 1 \mathrm{GeV}$. 1 Some hints are given, however, by DIS measurements in the non-perturbative region $Q<Q_{0}$. As illustrated in Fig. 1 of [3], the experimental constraints from DIS and DY are not given along a fixed value of $Q^{2}$ - as would be preferable for the DGLAP initial conditions but in certain kinematically correlated regions of $x$ and $Q^{2}$. Furthermore the data from DIS and DY are typically in distinct kinematical regions. For these reasons the input distributions must be constrained by using a recursive procedure similar to the global analyses of free parton distributions [23, 25].

In first approximation the input nuclear effects for valence and sea quarks can be assumed to be separately flavor-independent: $R_{u_{V}}^{A}\left(x, Q_{0}^{2}\right) \approx R_{d_{V}}^{A}\left(x, Q_{0}^{2}\right) \approx R_{V}^{A}\left(x, Q_{0}^{2}\right)$, and $R_{\bar{u}}^{A}\left(x, Q_{0}^{2}\right) \approx R_{\bar{d}}^{A}\left(x, Q_{0}^{2}\right) \approx R_{s}^{A}\left(x, Q_{0}^{2}\right)=R_{S}\left(x, Q_{0}^{2}\right)$ [3]. Thus only three independent input ratios must be constrained at $Q_{0}^{2}: R_{V}^{A}, R_{S}^{A}$ and $R_{G}^{A}$.

\section{Quarks and antiquarks}

- At $x \gtrsim 0.3$ the structure function $F_{2}^{A}$ is dominated by valence quark distributions. The DIS data for $R_{F_{2}}^{A}$ therefore only constrains the magnitude of the EMC effect and the Fermi-motion in $R_{V}^{A}$ but not those in $R_{S}^{A}$ or in $R_{G}^{A}$.

- At $0.04 \lesssim x \lesssim 0.3$ the DIS and DY data give bounds for $R_{S}^{A}$ and $R_{V}^{A}$ but in different regions of $Q^{2}$, (see Fig. 1 of [3]).

\footnotetext{
${ }^{7} \mathrm{In}$ [3] $Q_{0}=1.5 \mathrm{GeV}$ was chosen.
} 
- At $x \lesssim 0.04$ there are DIS data for the ratio $R_{F_{2}}^{A}$ available down to $x \sim 5 \cdot 10^{-3}$ in the region $Q \gtrsim 1 \mathrm{GeV}$ relevant for the DGLAP analysis. Conservation of baryon number forces the nuclear valence quarks to be less shadowed than the sea quarks.

- In the DIS data for $R_{F_{2}}^{A}$ at $x \lesssim 5 \cdot 10^{-3}$ one enters the nonperturbative region $Q \lesssim 1$ $\mathrm{GeV}$. A saturation behaviour of $R_{F_{2}}$ in $x \rightarrow 0$ is observed along the experimentally probed values of $Q^{2}$ [7, 11]. Provided that the sign of the slope of the $Q^{2}$ dependence of $R_{F_{2}}^{A}$ in the nonperturbative region remains the same (positive) as what is measured at $x \sim 0.01$ in the perturbative region [9], a saturation behaviour, i.e. a weak dependence of $R_{F_{2}}^{A}$ on $x$, can also be expected at $Q_{0}^{2}$. Constraints are then given by the DIS data in the non-perturbative region, in the sense that the data give a lower bound for $R_{F_{2}}^{A}\left(x, Q_{0}^{2}\right)$. Since at small values of $x$ the sea quark distributions dominate over the valence distributions, $R_{S}^{A}$ is also constrained by the DIS data while the shadowing in $R_{V}^{A}$ is restricted by baryon number conservation.

\section{Gluons and sea quarks}

- The scale dependence of $R_{F_{2}}^{A}$ at small values of $x$ is directly connected with shadowing of gluons: the more deeply gluons are shadowed, the slower is the evolution of $R_{F_{2}}^{A}$. The ratio $R_{G}^{A}$, can thus be constrained by the measured $Q^{2}$ dependence of $F_{2}^{A}$ as done in [19, 3]. Since the $Q^{2}$ dependence is not very strong, high-precision data is needed. In practice only the NMC data for $Q^{2}$ evolution of $F_{2}^{\mathrm{Sn}} / F_{2}^{\mathrm{C}}$ [9] can be used for constraining the input nuclear gluons at $0.02 \lesssim x \lesssim 0.2$.

- At $x \lesssim 0.02$ it can be assumed that $R_{G}^{A}\left(x, Q_{0}^{2}\right) \approx R_{F_{2}}^{A}\left(x, Q_{0}^{2}\right)$ for $x \ll 1$. This remains true within about $5 \%$ even after the DGLAP evolution from $Q_{0} \sim 1 \mathrm{GeV}$ to $Q \sim 100 \mathrm{GeV}$ [3].

- At $x \gtrsim 0.2$ there are no direct experimental constraints available but conservation of momentum together with arguments for stable evolution can be used. In this region one is sensitive to the small tails of the gluon distributions, and the existence of the EMC effect for nuclear gluons cannot be deduced based on the momentum sum rule alone. The evolution of gluon distributions are, however, affected by the valence quark distributions (but not vice versa), so since an EMC effect exists for the valence quarks, one will be generated for the gluons as well. The evolution equations for gluons and sea quarks are mutually coupled, so an EMC-like depletion will be generated for the sea quarks through the DGLAP evolution [2]. Then if the nuclear ratios $R_{i}^{A}$ do not move away from their input values very rapidly, it is a plausible first approximation to have an EMC effect both for the input gluon and sea quark distributions. For the sea quark modifications, which is the major subject of this paper, a simple assumption of $R_{S}\left(x \gtrsim 0.3, Q_{0}^{2}\right) \approx R_{V}\left(x \gtrsim 0.3, Q_{0}^{2}\right)$ 
was made in [3] for the input modifications of the sea quarks. We now move on to study the effects of this approximation on the Drell-Yan dilepton cross sections in nuclear collisions at SPS energies.

\section{Nuclear effects in DY production}

Nuclear effects in Drell-Yan dilepton production can be divided into two classes: first, there are "genuine" nuclear effects arising from the dynamics of the nuclear collision. These include nuclear modifications of parton distributions. Second, even without any nuclear effects in the parton densities or in the collision dynamics, the DY cross sections in $\mathrm{p}+A$ collisions (normalized per $A$ ) differ between isoscalar and non-isoscalar nuclei due to the different relative numbers of protons and neutrons. It is often convenient to use deuterium D as a reference, since it is approximately a sum of a free proton and a free neutron. Then any observed deviation of the DY cross section for $\mathrm{p}+A$ in isoscalar nuclei 114 from that for $\mathrm{p}+\mathrm{D}$ can be interpreted directly as a genuine nuclear effect, such as a nuclear modification in the parton distributions. For non-isoscalar nuclei, ratios of DY cross sections for $\mathrm{p}+A$ to those for $\mathrm{p}+\mathrm{D}$ or $\mathrm{p}+\mathrm{p}$ always show additional isospin effects.

For the purposes of comparison of the DY cross sections from $\mathrm{p}+A$ with those from $\mathrm{p}+\mathrm{D}$ and $\mathrm{p}+\mathrm{p}$, we write the isospin symmetric part of the parton distributions of the nucleons in a nucleus with $Z$ protons separately,

$$
Z f_{i}^{p / A}+(A-Z) f_{i}^{n / A}=\frac{A}{2}\left(f_{i}^{p / A}+f_{i}^{n / A}\right)+\left(Z-\frac{A}{2}\right)\left(f_{i}^{p / A}-f_{i}^{n / A}\right) .
$$

The inclusive cross section for the production of the Drell-Yan dilepton pairs of invariant mass $M$ and rapidity $y$ in $\mathrm{p}+A$ collisions can then be written in the lowest order as

$$
\begin{array}{r}
\frac{d \sigma_{D Y}^{p A}}{d M^{2} d y}=\frac{A}{2} \frac{8 \pi \alpha^{2}}{9 M^{2}} \frac{M^{2}}{s}\left\{\sum_{q=u, d, s, \ldots} e_{q}^{2}\left[q_{1}\left(\bar{q}_{2}^{p / A}+\bar{q}_{2}^{n / A}\right)+\bar{q}_{1}\left(q_{2}^{p / A}+q_{2}^{n / A}\right)\right]+\right. \\
\left.\left(\frac{2 Z}{A}-1\right) \sum_{q=u, d} e_{q}^{2}\left[q_{1}\left(\bar{q}_{2}^{p / A}-\bar{q}_{2}^{n / A}\right)+\bar{q}_{1}\left(q_{2}^{p / A}-q_{2}^{n / A}\right)\right]\right\},
\end{array}
$$

where $q_{i} \equiv f_{q}\left(x_{i}, Q^{2}\right)$ with the momentum fractions $x_{1,2}=\frac{M}{\sqrt{s}} \mathrm{e}^{ \pm y}$ and a scale choice $Q^{2}=M^{2}$. The ratio of the inclusive Drell-Yan cross section in a $\mathrm{p}+A$ collision vs. that in a $\mathrm{p}+\mathrm{p}$ or $\mathrm{p}+\mathrm{D}$ collision now becomes

$$
\begin{aligned}
& R_{D Y}^{A / B}\left(x_{2}, Q^{2}\right) \equiv \frac{\frac{1}{A} d \sigma_{D Y}^{p A} / d M^{2} d y}{\frac{1}{B} d \sigma_{D Y}^{p B} / d M^{2} d y} \\
& =\left\{4\left[u_{1}\left(\bar{u}_{2}^{A}+\bar{d}_{2}^{A}\right)+\bar{u}_{1}\left(u_{2}^{A}+d_{2}^{A}\right)\right]+\left[d_{1}\left(\bar{d}_{2}^{A}+\bar{u}_{2}^{A}\right)+\bar{d}_{1}\left(d_{2}^{A}+u_{2}^{A}\right)\right]+4 s_{1} s_{2}^{A}+\ldots\right\} / N_{D Y}^{B} \\
& +\left(\frac{2 Z}{A}-1\right)\left\{4\left[u_{1}\left(\bar{u}_{2}^{A}-\bar{d}_{2}^{A}\right)+\bar{u}_{1}\left(u_{2}^{A}-d_{2}^{A}\right)\right]+\left[d_{1}\left(\bar{d}_{2}^{A}-\bar{u}_{2}^{A}\right)+\bar{d}_{1}\left(d_{2}^{A}-u_{2}^{A}\right)\right]\right\} / N_{D Y}^{B}
\end{aligned}
$$


where the denominator $N_{D Y}^{B}$ only contains free parton densities. For deuterium $(B=$ $D=2) N_{D Y}^{B}$ is

$$
N_{D Y}^{D}=4\left[u_{1}\left(\bar{u}_{2}+\bar{d}_{2}\right)+\bar{u}_{1}\left(u_{2}+d_{2}\right)\right]+\left[d_{1}\left(\bar{d}_{2}+\bar{u}_{2}\right)+\bar{d}_{1}\left(d_{2}+u_{2}\right)\right]+4 s_{1} s_{2}+\ldots
$$

and, correspondingly, for the proton $(B=p=1)$ it is

$$
N_{D Y}^{p}=N_{D Y}^{D}+\sum_{q=u, d} 4\left[u_{1}\left(\bar{u}_{2}-\bar{d}_{2}\right)+\bar{u}_{1}\left(u_{2}-d_{2}\right)\right]+\left[d_{1}\left(\bar{d}_{2}-\bar{u}_{2}\right)+\bar{d}_{1}\left(d_{2}-u_{2}\right)\right] .
$$

It is evident that for isoscalar nuclei, $A=2 Z$, the ratio $R_{D Y}^{A / D}$ is unity in the absence of nuclear modifications in the parton densities. At large rapidities (large $\left.x_{F}\right) x_{2} \ll x_{1}$ and the ratio $R_{D Y}^{A / D}$ is sensitive mainly to the nuclear effects in the sea quark distributions. In the following, however, we are interested in the central rapidities $y \sim 0$, so $x_{1} \sim x_{2}$ and the ratio $R_{D Y}^{A / D}$ thus reflects the nuclear effects both in the sea quark and in the valence quark distributions.

For non-isoscalar nuclei on the other hand, even without nuclear modifications in the parton distribution functions, the isospin corrections $\sim\left(\frac{2 Z}{A}-1\right)$ must be considered. In fact, they depend quite strongly on the specific set of parton distributions used in the calculation. In the oldest sets, $\bar{u}=\bar{d}$ was assumed. Since $A \geq 2 Z$, this would lead to $R_{D Y}^{A / D}\left(x, Q^{2}\right) \leq 1$ in the absence of nuclear effects in the parton distributions. However, the ratio $\bar{u} / \bar{d}$ is experimentally different from unity: NA51 Collaboration measured this ratio for the first time, reporting a value of $\bar{u} / \bar{d}=0.51 \pm 0.04 \pm 0.05$ at $x=0.18$ [20]. This fact was taken into account in the subsequent sets of parton distributions, such as MRS94 [21], GRV94 222 and CTEQ94 [23]. Later on, E866 Collaboration at Fermilab measured this ratio with higher accuracy [24]. The modern parton distribution sets now include the $\bar{u} / \bar{d}$ asymmetry. As a result, the behaviour of the isospin corrections for the Drell-Yan process calculated by using the recent parton distribution sets differ from those calculated by using the old ones. To demonstrate this, we plot in Fig. 11 the ratio $R_{D Y}^{\mathrm{W} / D}$ computed with the parton densities of the free proton. For tungsten, $A=184$ and $Z=74$. The ratio is shown as a function of mass $M$ at $\sqrt{s}=30 \mathrm{GeV}$ and $y_{\mathrm{cm}}=0.04$ for four different sets of parton distributions.

Next, we study how the nuclear effects in the parton distributions and the isospin effects are reflected in the ratios $\frac{1}{A} \frac{d \sigma_{D Y}^{p A}}{d M} / \frac{1}{2} \frac{d \sigma_{D Y}^{p D}}{d M}$ and $\frac{1}{A} \frac{d \sigma_{D Y}^{p A}}{d M} / \frac{d \sigma_{D Y}^{p p}}{d M}$. These ratios can be formed from NA50 experimental results. They have measured inclusive dilepton production in $\mathrm{p}+\mathrm{p}, \mathrm{p}+\mathrm{D}, \mathrm{p}+{ }_{4}^{9} \mathrm{Be}$ and $\mathrm{p}+{ }_{74}^{184} \mathrm{~W}$ collisions at $E_{\mathrm{lab}}=450 \mathrm{GeV}(\sqrt{s}=30 \mathrm{GeV})$ at the CERN SPS in the rapidity range $3<y_{\text {lab }}<4\left(-0.46<y_{\mathrm{cm}}<0.54\right)$ and mass $M$ around the $J / \Psi$ peak. For $M \gtrsim 4 \mathrm{GeV}$, the mass spectrum is dominated by Drell-Yan dileptons.

For the following calculations, we integrate cross sections (5) over the NA50 rapidity bin, and form the above ratios. For the free parton distributions we use the set MRST98 


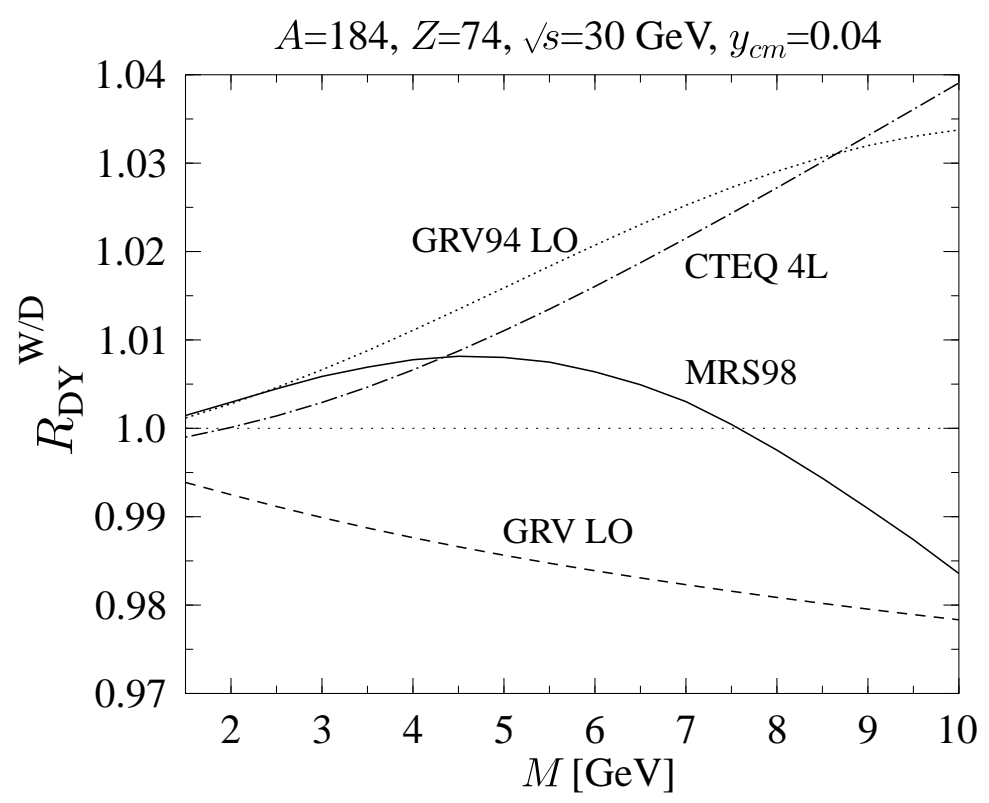

Figure 1: Isospin effects in the ratio of DY cross sections $R_{D Y}^{\mathrm{W} / \mathrm{D}}$ of Eq. (6) plotted as a function of mass $M$ for ${ }_{74}^{184} \mathrm{~W}$ at $\sqrt{s}=30 \mathrm{GeV}$ and $y_{\mathrm{cm}}=0.04$, computed with parton distributions from MRST98 (central gluon) [25] (solid), CTEQ4L [23] (dotted-dashed), GRV94LO [22] (dotted) and GRVLO [26] (dashed). Nuclear effects in the parton distributions are not included.

(central gluons) [25] and the nuclear effects in the parton distributions are taken from the EKS98 parametrization [4].

In Fig. 2 these ratios are plotted in lowest order for ${ }_{4}^{9} \mathrm{Be}$ (upper panel; solid, dashed) and ${ }_{74}^{184} \mathrm{~W}$ (lower panel; solid, dashed). The analysis [3] for the nuclear effects $R_{i}^{A}\left(x, Q^{2}\right)$ is only a leading order one, so strictly speaking it should be used only together with the leading order parton densities. The $Q^{2}$ evolution of the ratios $R_{i}^{A}\left(x, Q^{2}\right)$, however, is relatively slow, so the ratios given by EKS98 also serve as a first approximation for the nuclear effects in the next-to-leading-order (NLO) parton distributions. Keeping this source of uncertainty in mind, we have also computed the Drell-Yan cross sections in NLO [28. The ratios resulting from the NLO computation are also shown in Fig. 2 for ${ }_{4}^{9} \mathrm{Be}$ (upper panel; dotted-dashed, dotted) and ${ }_{74}^{184} \mathrm{~W}$ (lower panel; dotted-dashed, dotted). As seen in the figure, the LO ratios are a good approximation to the NLO ratios.

In NLO, $\mathcal{O}\left(\alpha^{2} \alpha_{s}\right)$, the Drell-Yan cross section consists of quark-antiquark annihilations with an emission of one real gluon; $q \bar{q}_{A}, \bar{q} q_{A} \rightarrow g \gamma^{*}$, and gluon-initiated Compton scatterings; $q g_{A}, g q_{A} \rightarrow q \gamma^{*}$ and $\bar{q} g_{A}, g \bar{q}_{A} \rightarrow \bar{q} \gamma^{*}$, and one-loop corrected quarkantiquark annihilations interfered with the LO annihilation. The total NLO contribution

\footnotetext{
${ }^{8}$ Fortran code from P.J. Rijken and W.L. van Neerven is used.
} 


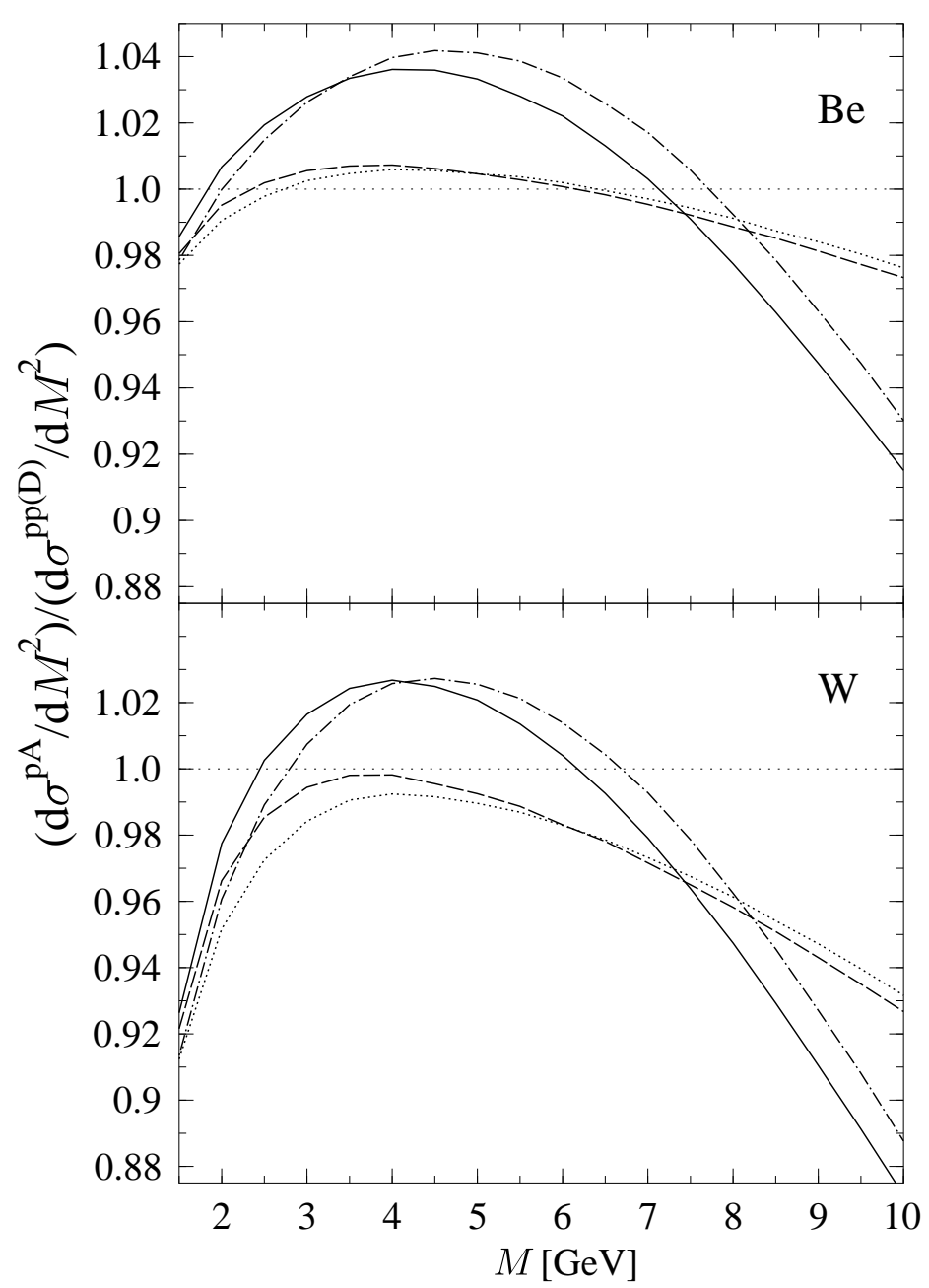

Figure 2: The $M$ dependence of the ratios of Drell-Yan cross sections $\frac{1}{A} \frac{d \sigma^{p A}}{d M}$ for $\mathrm{p}+A$-collisions at $\sqrt{s}=30 \mathrm{GeV}$ and $3<y_{\text {lab }}<4$. Upper panel: $\mathrm{p}+$ Be over $\mathrm{p}+\mathrm{p}$ for LO (solid) and for NLO (dotted-dashed), $\mathrm{p}+$ Be over $\mathrm{p}+\mathrm{D}$ for LO (dashed) and for NLO (dotted). Lower panel is the same for $\mathrm{p}+\mathrm{W}$. Shadowing and isospin corrections are taken into account

to $d \sigma / d M^{2} d y$ can be written as a sum $d \sigma_{N L O}=\sigma_{S+V}+\sigma_{H q \bar{q}}+\sigma_{g q}$, where $\sigma_{S+V}$ are the virtual corrections summed together with the soft emission of a gluon in the $q \bar{q}$ annihilations, $\sigma_{H q \bar{q}}$ contains the emissions of hard gluons in $q \bar{q}$ annihilations, and $\sigma_{g q}$ accounts for the Compton processes [28]. In the kinematical range studied here, $\sigma_{S+V}$ dominates the net NLO contribution. The sign of $\sigma_{H q \bar{q}}$ changes: $\sigma_{H q \bar{q}} / \sigma_{S+V} \sim+0.04 \ldots-0.39$ for $M=10 \ldots 1.5 \mathrm{GeV}$ and $y_{\mathrm{cm}}=0.04$. The Compton term $\sigma_{g q}$ remains negative and $\sigma_{g q} / \sigma_{S+V} \sim-0.12 \ldots-0.09$. Thus the net NLO effect is always a sum of partly canceling contributions and $K_{D Y}=\frac{d \sigma_{D Y}^{N L O}}{d M d y} / \frac{d \sigma_{D Y}^{L O}}{d M d y} \sim 1.6 \ldots 1.7$. In the ratios $R_{D Y}^{A / D}$ and $R_{D Y}^{A / p}$, however, 


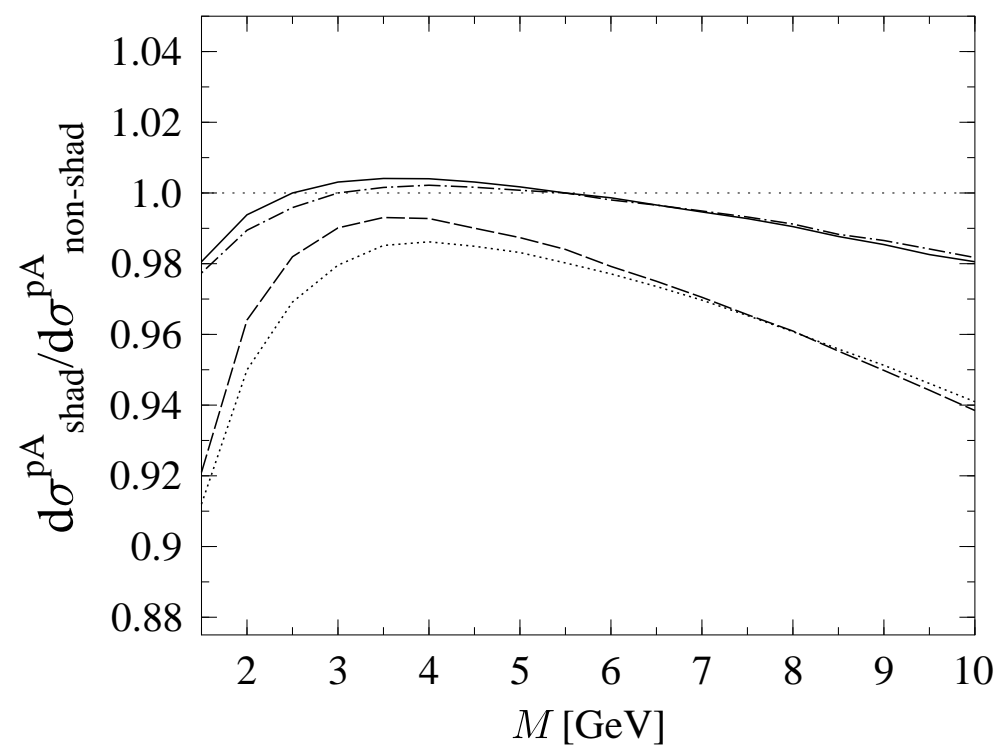

Figure 3: The ratio of shadowed over non-shadowed Drell-Yan cross sections $\frac{d \sigma^{p A}}{d M}$ for $\mathrm{p}+\mathrm{Be}$ LO (solid), p+Be NLO (dotted-dashed), $\mathrm{p}+\mathrm{W}$ LO (dashed) and $\mathrm{p}+\mathrm{W}$ NLO (dotted) collisions at $\sqrt{s}=30 \mathrm{GeV}$ and $3<y_{\text {lab }}<4$.

the $K$-factors largely cancel and the ratios remain very close to those computed in the LO, as seen in Fig. 2.

The ratios in Fig. 2 contain both the nuclear effects in the parton densities and the isospin corrections. To see the effect of the nuclear parton distributions alone, we have plotted in Fig. 3 the ratio of the cross sections $\frac{1}{A} \frac{d \sigma_{D Y}^{p A}}{d M}$ computed with and without nuclear effects in the parton distributions. Both the LO and NLO ratios are shown. The isospin corrections are taken into account in all cross sections; thus without nuclear modifications of the parton densities all the ratios in the figure would reduce to unity. By comparison with Fig. 2, and from Fig. 3, we conclude that the isospin corrections to the ratios $R_{D Y}^{A / D}$ remain small in magnitude when the MRS98 distributions are used.

Figs. 2 and 3 indicate that the net nuclear effects in DY caused by the nuclear modifications of parton densities are not very dramatic in $\mathrm{p}+A$ collisions at the SPS energy $E_{\text {lab }}=450 \mathrm{GeV} / c$ in the kinematic region $1 \mathrm{GeV} \lesssim M \lesssim 10 \mathrm{GeV}, 3<y_{\text {lab }}<4$. There are two reasons for this: first, at the corresponding values of $x_{2}$ the nuclear effects for the sea quarks are small. Second, since one is predominantly in the antishadowing region for valence quarks, there is a cancellation in the net nuclear effects in the ratio shown. To demonstrate this, and to show to what extent the ratio $\mathrm{p} A / \mathrm{pD}$ reflects the nuclear modifications of the sea and valence quarks, we show in Fig. 田 the nuclear effects 


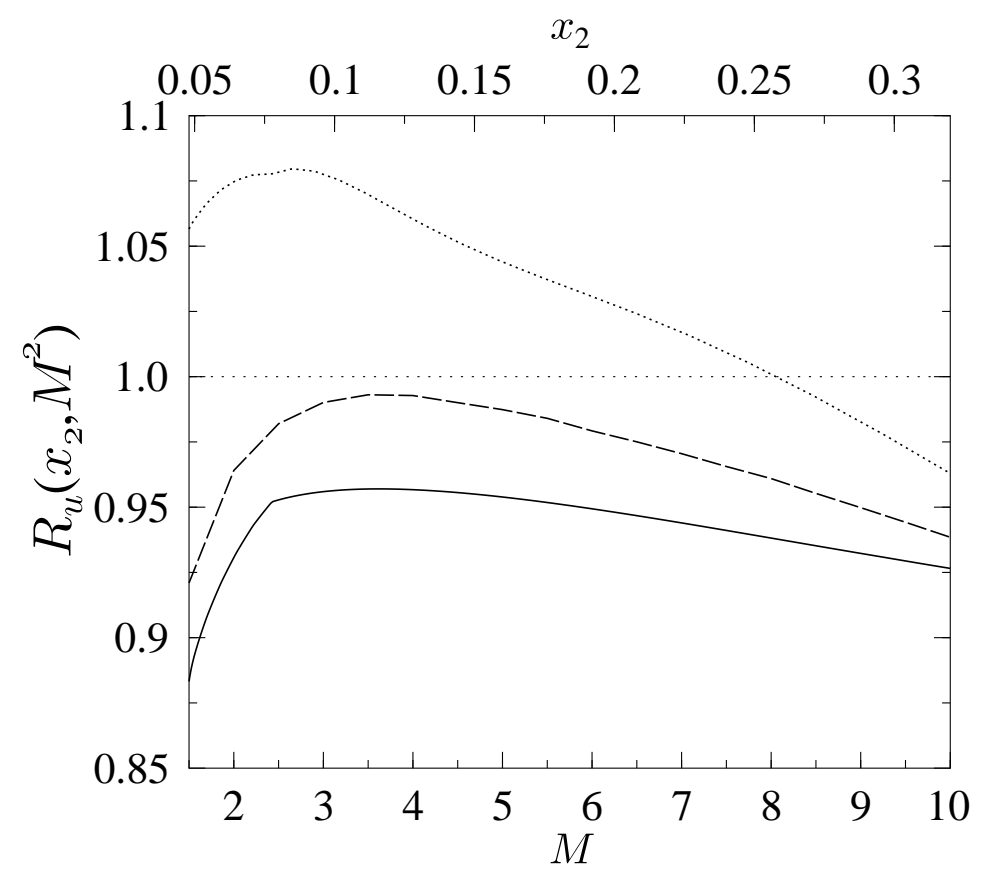

Figure 4: The ratios $R_{\bar{u}}^{A}\left(x_{2}, M^{2}\right)$ (solid) and $R_{u_{V}}^{A}\left(x_{2}, M^{2}\right)$ (dotted) for ${ }_{74}^{184} \mathrm{~W}$ as a function of invariant mass $M$ (lower $x$-axis) and $x_{2}=\frac{M}{\sqrt{s}} \mathrm{e}^{-y_{\mathrm{cm}}}$ (upper $x$-axis) for $\sqrt{s}=30 \mathrm{GeV}$ and $y_{\mathrm{cm}}=0.04$. The ratio of shadowed vs. non-shadowed LO DY cross sections (dashed) is the same as the dashed curve in Fig. 3.

$R_{u_{V}}^{A}\left(x_{2}, M^{2}\right)$ and $R_{\bar{u}}^{A}\left(x_{2}, M^{2}\right)$ as a function of mass $M$, with $x_{2}$ computed in the middle of the accepted rapidity bin (LO only), $x_{2}=\frac{M}{\sqrt{s}} \mathrm{e}^{-y_{\mathrm{cm}}}$ where $y_{\mathrm{cm}}=0.04$. The net effect in the $\mathrm{DY}$ ratio $\mathrm{pW} / \mathrm{pD}$ is shown again by the dashed curve. The values of $x_{2}$ can be read off from the top of the figure. Note here that the scale evolution of the sea quark modifications is taken into account.

To constrain the nuclear effects of the sea quarks at larger values of $x$, we must consider lower energies. The NA50 Collaboration at CERN has measured dilepton production in $\mathrm{Pb}+\mathrm{Pb}$ collisions at $E_{\text {lab }}=158 A \mathrm{GeV}(\sqrt{s}=17.2 \mathrm{GeV})$. This offers us a better chance for constraining the EMC effect in the input sea quark distributions. To illustrate the sensitivity of the DY dilepton cross sections $d \sigma^{\mathrm{Pb}+\mathrm{Pb}} / d M^{2}$ (LO, integrated over $\left.3<y_{\text {lab }}<4\right)$ to the assumption of the EMC effect in the input modifications of the nuclear sea quarks, the ratios

$$
\frac{d \sigma_{s h}^{\mathrm{Pb}+\mathrm{Pb}} / d M^{2}}{d \sigma^{\mathrm{Pb}+\mathrm{Pb}} / d M^{2}}
$$

are plotted in Fig. 5. The cross section $\frac{d \sigma^{\mathrm{Pb}+\mathrm{Pb}}}{d M^{2}}$ is the DY dilepton cross section which includes the isospin effects as in Eq. 1 but no nuclear effects of the parton distributions. 
The cross section $\frac{d \sigma_{s h}^{\mathrm{Pb}+\mathrm{Pb}}}{d M^{2}}$ similarly includes the isospin effects but is computed by using three different scenarios for the nuclear effects of the sea quarks. First we take into account all the nuclear effects as given by the EKS98. The resulting ratio is shown by the solid curve in Fig. 5. Second, we keep the nuclear effects of the valence quarks in accordance with EKS98 but relax the assumption of the EMC-effect in the sea component. We take the ratios $R_{\bar{q}}^{A}\left(=R_{q_{S}}^{A}\right.$ ) from EKS98 at $x<0.1$ (where these ratios are constrained by experimental data) but interpolate $R_{\bar{q}}^{A}$ linearly from $x=0.3$ to the region of Fermi motion $x \gtrsim 0.8$ without an EMC effectfy. The resulting ratio is shown by the dashed curve in Fig. 5. Finally, we switch off all nuclear effects in the sea quarks and antiquarks by setting $R_{\bar{q}}^{A}=1$. We emphasize that the last scenario is actually unphysical as it violates the available constraints, and that it is meant only for comparison purposes, to see the effects of the nuclear corrections in valence quarks alone. The corresponding ratio is shown by the dotted curve. Note that the deviation from unity of the ratios in Fig. 5 directly shows the effects of the nuclear modifications in the parton distributions. The net effect of nuclear parton distributions is now clearly larger than in the $\mathrm{p}+A$ case.

In principle it should be possible to form this ratio from the measurements, by taking the numerator directly from $\mathrm{Pb}+\mathrm{Pb}$ data and the denominator from $\mathrm{p}+\mathrm{p}$ and $\mathrm{p}+\mathrm{D}$ data. To our knowledge, however, no experimental data presently exists for Drell-Yan production in $\mathrm{p}+\mathrm{p}$ or $\mathrm{p}+\mathrm{D}$ collisions at $E_{\text {lab }}=158 \mathrm{AGeV} / \mathrm{c}$. Thus, some additional input is needed in order to form the experimental ratio (9). One possibility is to compare the measured $\mathrm{DY}$ cross section in $\mathrm{Pb}+\mathrm{Pb}$ directly with a purely theoretical calculation. Alternatively, the denominator in Eq. (9) could be formed from other $\mathrm{p}+\mathrm{p}$ or $\mathrm{p}+\mathrm{D}$ data (e.g. data from NA51 collaboration at $E_{\text {lab }}=450 \mathrm{GeV} / c$ 20]) corrected to $E_{\text {lab }}=$ $158 \mathrm{AGeV} / c$, based on theoretical cross sections without nuclear effects in the parton distributions. In both cases modern parton distributions, in which constraints from the measured ratio $\bar{u} / \bar{d}$ are included, must to be used in order to correctly include isospin corrections. The main uncertainty would be the overall normalization, which is common to both cases. As shown by Fig. 5, the experimental ratio is expected to be unity within $\mathrm{a} \sim 5 \%$ uncertainty at $M \simeq 4 \mathrm{GeV}$. If a direct measurement could be made at a single $M$-value, it could be used to fix the overall normalization of the ratio. Then the slope of the ratio towards larger values of $M$ would give direct information of the EMC effect in the sea quark distributions.

Our study shows that the expected nuclear effects are $\gtrsim 20 \%$ at masses larger than 7 $\mathrm{GeV}$. Relevant constraints would be obtained for the EMC effect of the sea quarks and antiquarks if the precision is $\sim 10 \%$.

\footnotetext{
${ }^{9}$ This procedure would cause some inconsistency with the EKS98-modification of gluons due to the scale evolution of the parton densities but as we now do the DY computation in the LO only, gluons are not involved.
} 


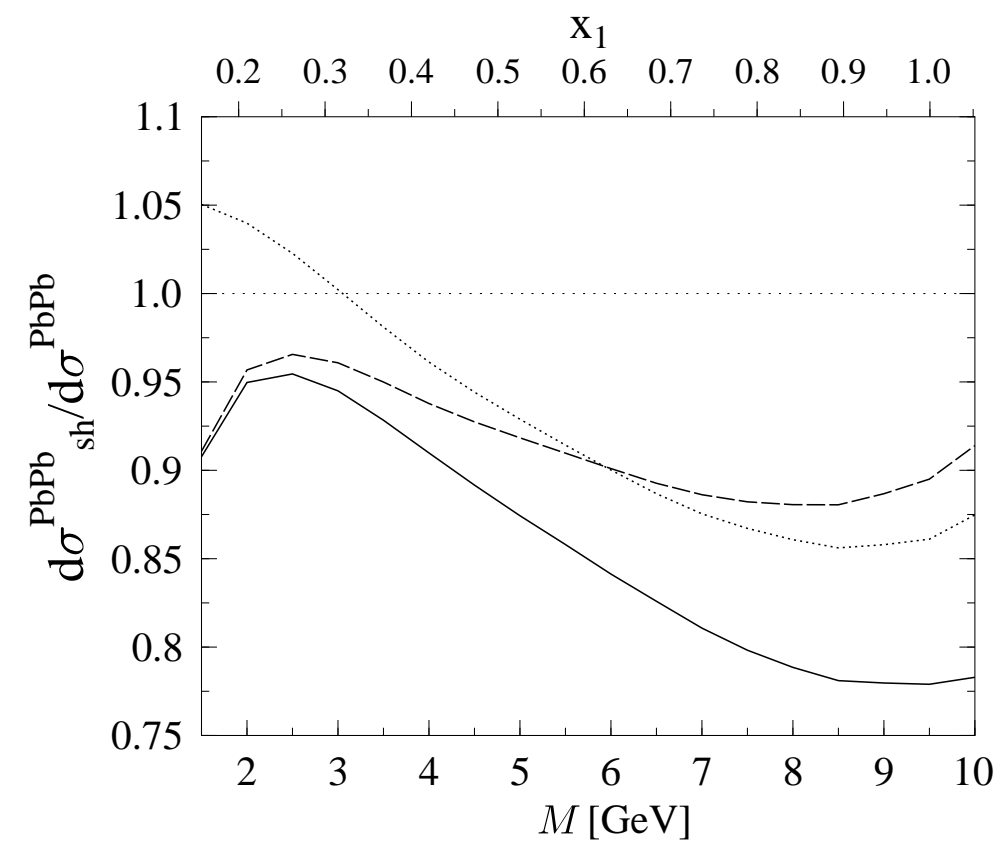

Figure 5: The ratio (9) of the distributions $d \sigma / d M^{2}$ at $3<y_{\text {lab }}<4$ computed with and without the nuclear effects in parton distributions for $\mathrm{Pb}+\mathrm{Pb}$ collisions at $E_{\text {lab }}=158 \mathrm{AGeV}$. Solid curve: $R_{\bar{q}}^{A}$ taken from the EKS98 parametrization. Dashed curve: $R_{\bar{q}}^{A}$ interpolated over the region $0.1<x<0.85$ by assuming no EMC effect. Dotted curve: $R_{\bar{q}}^{A}=1$. Isospin effects are included. The values of $x_{2}=\frac{M}{\sqrt{s}} \mathrm{e}^{-y_{\mathrm{cm}}}$ and $x_{1}=\frac{M}{\sqrt{s}} \mathrm{e}^{y_{\mathrm{cm}}}$, computed in the middle of the $y$ bin can be read off from the upper $x$ axis.

\section{Conclusions}

We have studied the sensitivity of the Drell-Yan cross sections in SPS nuclear collisions to isospin corrections and to the nuclear modifications of the parton densities in the regions of antishadowing and EMC effect. Our aim has been to find a way to constrain the nuclear modifications of the sea quark distributions in order to improve the determination of the input modifications in the DGLAP analysis [3] of nuclear parton distributions. We have shown that the Drell-Yan dilepton data collected by the NA50 collaboration at CERN $\mathrm{SPS}$ in $\mathrm{Pb}+\mathrm{Pb}$ collisions at $E_{\text {lab }}=158 \mathrm{AGeV} / \mathrm{c}$ would be suitable for constraining the EMC effect in the input distributions of the sea quarks provided that a sufficient precision, $\sim 5 \ldots 10 \%$ is reached in forming the ratio $\frac{d \sigma_{s h}^{\mathrm{Pb}+\mathrm{Pb}}}{d M^{2}} / \frac{d \sigma^{\mathrm{Pb}+\mathrm{Pb}}}{d M^{2}}$ at $M \gtrsim 5 \mathrm{GeV}$.

We have also shown that for $\mathrm{p}+A$ collisions at $E_{\mathrm{lab}}=450 \mathrm{GeV}$, in the kinematic range $3<y_{\text {lab }}<4$, the net effects due to nuclear modifications of the parton densities are small. This is because typical values of $x$ remain in the region where the nuclear effects in the sea quarks and valence quarks largely cancel. Even at the highest masses studied, $M \sim 10$, where the typical $x$ for $E_{\text {lab }}=450 \mathrm{GeV}$ is $\sim 0.3$, the nuclear parton distributions modify 
the $\mathrm{p}+\mathrm{W}$ cross sections only by $6 \%$. This sets the minimum precision required for such an experiment to constrain the nuclear sea quark distributions in the antishadowing region. We have also shown that the isospin effects are small, provided that modern parton distributions, where $\bar{u} \neq \bar{d}$, are used.

Based on the data from $\mathrm{p}+A$ collisions, it is often assumed that Drell-Yan behaves as $A^{1}$ in nuclear collisions. From our results for the SPS, Figs. 2 and 3, we see that strictly speaking this is not the case but the deviations remain fairly small, and within a $5 \%$ uncertainty in the cross sections the deviations can be neglected. However, at higher energies, such as $E_{\text {lab }}=800 \mathrm{GeV}$ in the Fermilab E772 experiments, the shadowing corrections at smaller values of $x$ become important and have been experimentally observed [14, 15.

Finally, let us comment on the consequences of these nuclear effects in the analysis of the Drell-Yan cross sections in $\mathrm{Pb}+\mathrm{Pb}$ collisions measured by the NA50 collaboration in connection with $J / \Psi$ suppression [18]. We have shown that the slope of the invariant mass distributions of the DY pairs is affected by nuclear effects: Fig. 0 indicates that corrections of the order of $20 \%$ appear at $M \sim 7 . .8 \mathrm{GeV}$ (assuming the EKS98 modifications) but below the $J / \Psi$ peak they are only about $5 \%$. The data points at large masses, however, have a smaller weight in the $\chi^{2}$ fits [18 due to relatively large statistical uncertainties. The fits are dominated by pair masses near $4 \mathrm{GeV}$ where the error bars are smaller. The nuclear effects in the mass distributions thus remain less than $5 \%$ for the extrapolation of the DY cross sections from $4 \mathrm{GeV}$ down to $3 \mathrm{GeV}$. On

the other hand, the experimental $K$-factor, $K=\sigma_{\exp }^{\mathrm{DY}} / \sigma_{\mathrm{th}}^{\mathrm{DY}, \mathrm{LO}} 18$ includes the region of large M, and thus could be underestimated by $10 . .15 \%$ if the nuclear effects in parton distributions are neglected.

\section{Acknowledgements.}

We thank C. Lourenço, V. Ruuskanen and Yu.M. Shabelski for useful discussions. CAS thanks Ministerio de Educación y Ciencia of Spain for financial support. This work was supported by the Academy of Finland, grant no. 42376.

\section{References}

[1] Yu. Dokshitzer, Sov. Phys. JETP 46 (1977) 1649; V.N. Gribov and L. N. Lipatov, Sov. Nucl. Phys. 15 (1972) 438, 675; G. Altarelli, G. Parisi, Nucl. Phys. B126 (1977) 298.

[2] K.J. Eskola, Nucl. Phys. B400 (1993) 240.

[3] K.J. Eskola, V.J. Kolhinen and P.V. Ruuskanen, Nucl. Phys. B535 (1998) 351.

[4] K.J. Eskola, V.J. Kolhinen and C.A. Salgado, Eur. Phys. J. C 9 (1999) 61. 
[5] EMC collaboration, J.J. Aubert et al., Phys. Lett. B123 (1983) 275; A. Bodek et al., Phys. Rev. Lett. 50 (1983) 1431; 51 (1983) 534.

[6] NMC collaboration, P. Amaudruz et al., Nucl. Phys. B441 (1995) 3.

[7] NMC collaboration, M. Arneodo et al., Nucl. Phys. B441 (1995) 12.

[8] NMC collaboration, M. Arneodo et al., Nucl. Phys. B481 (1996) 3.

[9] NMC collaboration, M. Arneodo et al., Nucl. Phys. B481 (1996) 23.

[10] J. Gomez et al., Phys. Rev. D49 (1994) 4348.

[11] E665 collaboration, M.R. Adams et al., Phys. Rev. Lett. 68 (1992) 3266.

[12] E665 collaboration, M.R. Adams et al., Z. Phys. C67 (1995) 403.

[13] M. Arneodo, Phys. Rep. 240 (1994) 301.

[14] D. M. Alde et al., Phys. Rev. Lett. 64 (1990) 2479.

[15] E866 Collaboration, M.A. Vasiliev et al., Phys. Rev. Lett. 83 (1999) 2304.

[16] http://urhic.phys.jyu.fi/, http://www-fp.usc.es/phenom

[17] H. Plothow-Besch, Int. J. Mod. Phys. A10 (1995) 2901; Comput. Phys. Comm. 75 (1993) 396; PDFLIB User's Manual - Version 8.04, W5051 PDFLIB, 2000.04.17, CERN-ETT/TT.

[18] NA50 Collaboration, M.C. Abreu et al., Phys. Lett. B410 (1997) 337.

[19] T. Gousset and H.J. Pirner, Phys. Lett. B375 (1996) 349.

[20] NA51 collaboration, A. Baldit et al., Phys. Lett. B332 (1994) 244.

[21] A.D. Martin, W.J. Stirling and R.G. Roberts, Phys. Rev. D50 (1994) 6734; Phys. Rev. D51 (1995) 4756.

[22] M. Glück, E. Reya and A. Vogt, Z. Phys. C67 (1995) 433.

[23] H.L. Lai et al., Phys. Rev. D55 (1997) 1280.

[24] E866 collaboration, E.A. Hawker et al., Phys. Rev. Lett. 80, (1998) 3715.

[25] A.D. Martin, R.G. Roberts, W.J. Stirling, R.S. Thorne, Eur. Phys. J. C4 (1998) 463. 
[26] M. Glück, E. Reya and A. Vogt, Z. Phys. C53 (1992) 127.

[27] NA38 Collaboration, M.C. Abreu et al. Phys. Lett. B423 (1998) 207.

[28] P.J. Rijken and W.L. van Neerven, Phys. Rev. D51 (1995) 44. 